First publ. in: Physical Review B, Vol. 70 (2004), Article 020502

\title{
Kondo effect and Josephson current through a quantum dot between two superconductors
}

\author{
Mahn-Soo Choi, ${ }^{1}$ Minchul Lee, ${ }^{1}$ Kicheon Kang, ${ }^{2}$ and W. Belzig ${ }^{3}$ \\ ${ }^{1}$ Department of Physics, Korea University, Seoul 136-701, Korea \\ ${ }^{2}$ Department of Physics and Institute for Condensed Matter Theory, Chonnam National University, Kwangju 500-757, Korea \\ ${ }^{3}$ Department of Physics and Astronomy, University of Basel, Klingelbergstrasse 82, 4056 Basel, Switzerland
}

(Received 12 May 2004; published 9 July 2004)

\begin{abstract}
We investigate the supercurrent through a quantum dot for the whole range of couplings using the numerical renormalization group method. We find that the Josephson current switches abruptly from a $\pi$ - to a 0-phase as the coupling increases. At intermediate couplings the total spin in the ground state depends on the phase difference between the two superconductors. Our numerical results can explain the crossover in the conductance observed experimentally by Buitelaar et al. [Phys. Rev. Lett. 89, 256801 (2002)].
\end{abstract}

PACS number(s): 74.50. $+\mathrm{r}, 72.15 . \mathrm{Qm}, 75.20 . \mathrm{Hr}$

\section{INTRODUCTION}

The Kondo effect and superconductivity are two of the most extensively studied phenomena in condensed matter physics ever since the pioneering works by Kondo ${ }^{1}$ and by Bardeen, Cooper and Schrieffer, ${ }^{2}$ respectively. When a localized spin is coupled to superconducting electrons, the two effects are intermingled and even richer physics will emerge. The physically interesting questions are: Would the Kondo effect survive, overcoming the spin-singlet pairing of electrons in superconductors ( $\mathrm{SCs}$ ) and the superconducting gap at the Fermi level? If it does, how would such a strongly correlated state affect the transport, especially the Josephson current, between two superconductors?

The Josephson effect through a strongly interacting region with a localized spin was discussed long before by Shiba and Soda $^{3}$ and Glazman and Matveev ${ }^{4}$ and further elucidated by Spivak and Kivelson..$^{5}$ The large on-site interaction only allows the electrons in a Cooper pair to tunnel one by one via virtual processes in which the spin ordering of the Cooper pair is reversed, leading to a negative Josephson coupling (i.e., a $\pi$-junction). This argument, however, is based on a perturbative idea and holds true only for sufficiently weak tunneling. It was suggested ${ }^{4}$ that as the tunneling increases, the Kondo effect produces a collective resonance at the Fermi level. As a result, the Josephson current is enhanced by the Coulomb repulsion. Moreover, the Josephson coupling is expected to be positive (i.e., a 0 -junction) since the localized spin is screened due to the Kondo effect. Based on this, Glazman and Matveev ${ }^{4}$ assumed a strong coupling fixed point and derived the Josephson current as a function of phase difference. Recently, several approximation methods have been used to investigate the transition from the 0 - to $\pi$-junction as a function of the tunneling strength: ${ }^{6-9}$ A modified Hartree-Fock approximation, ${ }^{6}$ a non-crossing approximation, ${ }^{7}$ and a variational method $^{8}$ predict a $0-\pi$ transition, whereas the slave-boson mean-field theory ${ }^{8}$ always favors the Kondo effect.

In this work, we use a numerical renormalization group (NRG) method to investigate thoroughly the $0-\pi$ transition as well as to examine the argument above suggested by Glazman and Matveev. ${ }^{4}$ Based on the NRG method, we calculate quantitatively the local properties (i.e., the pairing cor- relation and the single-particle excitation spectrum) of the quantum dot (QD), the total spin in the ground-state wave function, and the Josephson current as a function of phase difference. Finally, we show that our numerical results can explain the experimentally observed crossover of the conductance in SC-carbon nanotube-SC junctions. ${ }^{10}$

\section{MODEL}

The system consists of a QD with an odd number of electrons coupled to two superconducting leads ( $L$ and $R$ ). The study of Kondo effect in such a mesoscopic system has recently attracted much interest due to its tunability. As already demonstrated experimentally with normal leads, ${ }^{11}$ it allows for various tests of Kondo physics, which are difficult in bulk solids. The two leads are regarded to be standard $s$-wave superconductors (SCs) and described by the BCS Hamiltonian

$$
\begin{aligned}
\mathcal{H}_{\mathrm{BCS}}= & \sum_{\ell=L, R} \sum_{\mathbf{k}, \sigma} \epsilon_{\ell, \mathbf{k}} c_{\ell, \mathbf{k}, \sigma}^{\dagger} c_{\ell, \mathbf{k} \sigma}-\sum_{\ell} \sum_{\mathbf{k}}\left(\Delta_{\ell} e^{+i \phi_{\ell}} c_{\ell, \mathbf{k}, \uparrow}^{\dagger} c_{\ell,-\mathbf{k}, \downarrow}^{\dagger}\right. \\
& + \text { h.c. }),
\end{aligned}
$$

where $c_{\ell, \mathbf{k}, \sigma}^{\dagger}\left(c_{\ell, \mathbf{k}, \sigma}\right)$ creates (destroys) an electron with energy $\epsilon_{\ell, \mathbf{k}}$, momentum $\hbar \mathbf{k}$, and spin $\sigma$ on the lead $\ell . \Delta_{\ell}$ is the superconducting gap and $\phi_{\ell}$ is the phase of the superconducting order parameter. The QD is described by an Anderson-type impurity model

$$
\mathcal{H}_{\mathrm{QD}}=\sum_{\sigma} \epsilon_{d} d_{\sigma}^{\dagger} d_{\sigma}+U d_{\uparrow}^{\dagger} d_{\uparrow} d_{\downarrow}^{\dagger} d_{\downarrow}
$$

which is widely adopted for sufficiently small quantum dots. In Eq. (2) $d_{\sigma}^{\dagger}$ and $d_{\sigma}$ are electron creation and annihilation operators on the QD. The level position $\epsilon_{d}$, measured from the Fermi energy $E_{F}$ of the two leads (throughout the paper every energy is measured from $E_{F}$ ), can be tuned by an external gate voltage. The interaction $U$ is order of charging energy $e^{2} / 2 C$ ( $C$ is the capacitance of the QD). The coupling between the QD and the SCs is described by the tunneling Hamiltonian 


$$
\mathcal{H}_{V}=\sum_{\ell} \sum_{\mathbf{k}, \sigma} V_{\ell}\left(d_{\sigma}^{\dagger} c_{\ell, \mathbf{k}, \sigma}+\text { h.c. }\right)
$$

Putting all together the Hamiltonian for the whole system is given by $\mathcal{H}=\mathcal{H}_{\mathrm{QD}}+\mathcal{H}_{\mathrm{BCS}}+\mathcal{H}_{V}$.

We take a few simplifications to make clearer the physical interpretation of the results below. The two SCs are assumed to be identical $\left(\epsilon_{L, \mathbf{k}}=\epsilon_{R, \mathbf{k}}=\epsilon_{\mathbf{k}}\right.$ and $\left.\Delta_{L}=\Delta_{R}=\Delta\right)$ except for a finite phase difference $\phi=\phi_{L}-\phi_{R}$; without loss of generality we put $\phi_{L}=-\phi_{R}=\phi / 2$. In the normal state, the conduction bands on the leads are symmetric with a flat density of states $N_{0}$ and the width $D$ above and below the Fermi energy. We also put $\epsilon_{d}=-U / 2$ in $\mathcal{H}_{Q D}$, Eq. (2); it has been checked that an asymmetric model $\left(\epsilon_{d} \neq-U / 2\right)$ gives the qualitatively same results for physical quantities of our concern. We only consider the symmetric junction, $V_{L}=V_{R}=V$. The coupling to the leads is well characterized by the single parameter $\Gamma$ $=2 \pi N_{0} V .^{2}$ Below we will distinguish the strong $\left(T_{K} \gg \Delta\right)$ and the weak $\left(T_{K} \ll \Delta\right)$ coupling limits by the ratio between the superconducting gap $\Delta$ and the normal-state Kondo temperature $T_{K}\left(k_{B}=1\right)$ given by ${ }^{12}$

$$
T_{K}=\Gamma \sqrt{\frac{U}{2 \Gamma}} \exp \left[\pi \frac{\epsilon_{d}}{2 \Gamma}\left(1+\frac{\epsilon_{d}}{U}\right)\right] .
$$

Following the standard NRG procedures ${ }^{13,14}$ extended to superconducting leads, ${ }^{15}$ we evaluate the various physical quantities from the recursion relation

$$
\begin{aligned}
\tilde{\mathcal{H}}_{N+1}= & \sqrt{\Lambda} \tilde{\mathcal{H}}_{N}+\xi_{N} \sum_{\mu, \sigma}\left(f_{\mu, N, \sigma}^{\dagger} f_{\mu, N+1, \sigma}+\text { h.c. }\right) \\
& -\Lambda^{N / 2} \sum_{\mu} \widetilde{\Delta}_{\mu}\left(f_{\mu, N+1, \uparrow}^{\dagger} f_{\mu, N+1, \downarrow}^{\dagger}+\text { h.c. }\right)
\end{aligned}
$$

with the initial Hamiltonian given by

$$
\begin{aligned}
\tilde{\mathcal{H}}_{0}= & \frac{1}{\sqrt{\Lambda}}\left[\tilde{\mathcal{H}}_{\mathrm{QD}}+\sum_{\mu=e, o} \sum_{\sigma} \tilde{V}_{\mu}\left(d_{\sigma}^{\dagger} f_{\mu, 0, \sigma}+\text { h.c. }\right)\right. \\
& \left.-\sum_{\mu} \widetilde{\Delta}_{\mu}\left(f_{\mu, 0, \uparrow}^{\dagger} f_{\mu, 0, \downarrow}^{\dagger}+\text { h.c. }\right)\right] .
\end{aligned}
$$

Here the fermion operators $f_{\mu, N, \sigma}$ have been introduced as a result of the logarithmic discretization and the accompanying canonical transformation, $\Lambda$ is the logarithmic discretization parameter (we choose $\Lambda=2$ ), $\xi_{N} \sim 1,{ }^{13}$ and

$$
\begin{gathered}
\tilde{\mathcal{H}}_{\mathrm{QD}} \equiv \zeta \frac{\mathcal{H}_{\mathrm{QD}}}{D}, \quad \tilde{\Delta}_{\mu} \equiv \zeta \frac{\Delta_{\mu}}{D}, \\
\tilde{V}_{e} \equiv \zeta \sqrt{\frac{2 \Gamma}{\pi D}} \cos (\phi / 4), \quad \tilde{V}_{o} \equiv-\zeta \sqrt{\frac{2 \Gamma}{\pi D}} \sin (\phi / 4),
\end{gathered}
$$

with $\zeta=2 /(1+1 / \Lambda)$. The Hamiltonians $\widetilde{\mathcal{H}}_{N}$ in Eq. (5) have been rescaled for numerical accuracy. The original Hamiltonian is recovered by $\mathcal{H} / D=\lim _{N \rightarrow \infty} \widetilde{\mathcal{H}}_{N} / \mathcal{J}_{N}$ with $\mathcal{J}_{N}$ $\equiv \zeta \Lambda^{(N-1) / 2}$.

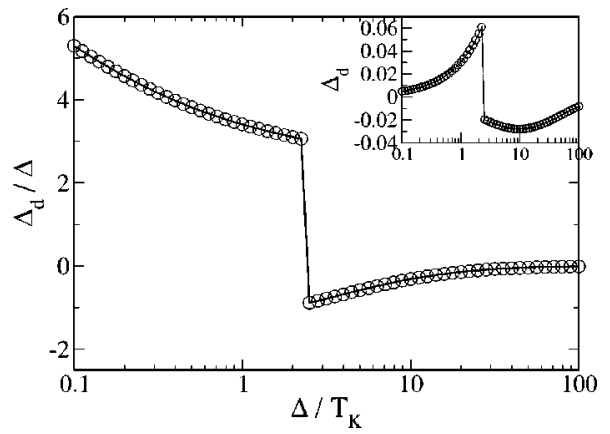

FIG. 1. The pairing correlation on the quantum dot, $\Delta_{d}$ $\equiv\left\langle d_{\uparrow}^{\dagger} d_{\downarrow}^{\dagger}\right\rangle$, as a function of $\Delta / T_{K}$. Inset: plot of bare (not normalized) values of $\Delta_{d}$. We have chosen $\epsilon_{d}=-U / 2=-0.1 D$ and $\Gamma=0.04 D$.

\section{PROXIMITY EFFECT}

To see how superconductivity on the leads affects the interacting QD in the strong and weak coupling limits, we first examine the local properties on the QD with zero phase difference $(\phi=0) .{ }^{16}$ Figure 1 shows the local pair correlation $\Delta_{d} \equiv\left\langle d_{\uparrow}^{\dagger} d_{\downarrow}^{\dagger}\right\rangle$ as a function of $\Delta / T_{K}$. As expected, the local pair correlation $\Delta_{d}$ vanishes with $\Delta$, and gets smaller (even vanishes when $U \rightarrow \infty$ ) as $\Delta \rightarrow \infty$; see Fig. 1 (inset). An interesting aspect of $\Delta_{d}$ is the sign change at $\Delta=\Delta_{c} \simeq 2.4 T_{K}$, which suggests that the physical properties are different in the strong $\left(T_{K} \gg \Delta\right)$ and the weak $\left(T_{K} \ll \Delta\right)$ coupling limits. Indeed we see (from the NRG calculation) that the groundstate wave function of the whole system is of spin singlet (the localized spin is screened out) for $\Delta<\Delta_{c}$ and of spin doublet (the SCs form Cooper pairs separately and the localized spin is left unscreened) for $\Delta>\Delta_{c}$. The negative sign in $\Delta_{d}$ in the weak coupling limit can be explained by a simple second-order perturbation theory, while the positive one in the strong-coupling limit is expected when there is a resonance channel for Cooper-pair tunneling. ${ }^{17}$ Therefore, it seems quite plausible to argue that in the strong coupling limit the Kondo resonance develops even in the presence of the superconducting gap in the conduction band and the proximity effect arises through the resonance; see also the discussion of the Josephson current below. Putting it another way, the local moment of spin $1 / 2$ induces a negative $\Delta_{d}$ for weak couplings, but as the coupling increases it is screened and a positive $\Delta_{d}$ is recovered.

This interpretation is further supported by the singleparticle excitation spectra $A_{d}(E)$ on the QD, as shown in Fig. 2 for different values of $\Delta / T_{K}$. In Fig. 2(a) $A(E)$ for zero phase difference ${ }^{18}$ is shown and we observe a qualitative change of the spectrum when $\Delta$ becomes smaller than $T_{K}$. A localized state below the superconducting gap appears for $\Delta \gtrsim T_{K}$, whereas the spectrum has a gap of the order of $\Delta$ in the other limit. The other panels in Fig. 2 show the phasedependent density of states in the sub-gap regime. We clearly observe a phase-dependent formation of an Andreev bound state. For $\Delta / T_{K}=0.1$ the Andreev state emerges from the gap with increasing phase and reaches the smallest energy for $\phi=\pi$, which is reminiscent of a usual superconducting junction. In weak coupling limit, $\Delta=10 T_{K}$, we observe an opposite phase-dependence, which is similar to the predicted 


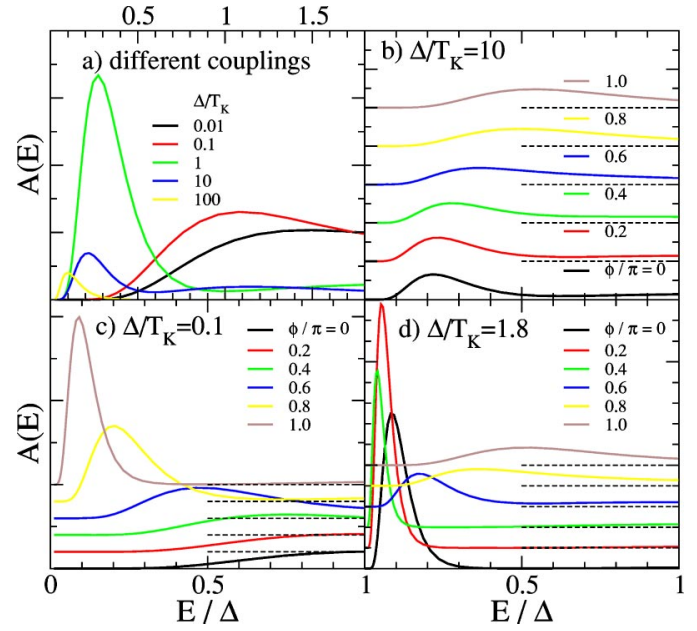

FIG. 2. (Color online) The single-particle excitation spectrum on the quantum dot. $\epsilon_{d}=-U / 2=-0.1 D$ and $\Gamma=0.04 D\left(T_{K}\right.$ $=0.0089 D$ ).

$\pi$-junction behavior. ${ }^{4}$ For an intermediate coupling, $\Delta / T_{K}$ $=1.8$, there is always a localized state below the gap, which has a non-monotonic phase-dependence. In the following, we will discuss the Josephson current through the quantum dot.

\section{JOSEPHSON CURRENT}

We now turn to the Josephson current through the QD in the presence of a finite phase difference $\phi$. Within the NRG method, the Josephson current can be conveniently calculated by ${ }^{19}$

$$
\frac{I_{S}(\phi)}{I_{c}^{\text {short }}}=-\sqrt{\frac{D \Gamma}{2 \pi \Delta^{2}}}\left[\sin (\phi / 4) J_{e}+\cos (\phi / 4) J_{o}\right]
$$

with $J_{\mu} \equiv \Sigma_{\sigma}\left(d_{\sigma}^{\dagger} f_{\mu, 0, \sigma}+\right.$ h.c. $)(\mu=e, o)$. Here $I_{c}^{\text {short }} \equiv e \Delta / \hbar$ is the critical current of a transparent single-mode junction. ${ }^{17}$

Figure 3 shows the Josephson current as a function of phase difference $\phi$ between the two superconducting leads for different values of ratio $\Delta / T_{K}$. In the weak coupling limit $\left(T_{K} \ll \Delta\right)$, it is clearly seen from Fig. 3(a) that the effective Josephson coupling is negative (i.e., a $\pi$ - junction). ${ }^{3-5,7,20} \mathrm{In}$ addition, the supercurrent-phase relation is very close to a sinusoidal function, like typical "tunneling junctions." $17 \mathrm{We}$ also report that the ground state is a doublet for any phase difference $\phi$.

In the strong coupling limit $\left(T_{K} \gg \Delta\right)$, on the other hand, the Josephson coupling is positive $;^{6-8}$ see Fig. 3(b). Another remarkable thing is that the current-phase relation is highly non-sinusoidal and reminiscent of the current-phase relation in the short junction limit. ${ }^{17}$ Furthermore, the critical current approaches the unitary limit $I_{c}^{\text {short }}$ of "short junctions"17 as the coupling grows stronger $\left(\Delta / T_{K} \rightarrow 0\right)$, as shown in Fig. 3(d). These results suggest again that in the strong coupling limit the Kondo resonance develops at the Fermi level and Cooper pairs tunnel resonantly through it. Naturally, the ground state turns out to be a spin singlet for any $\phi$. It should be stressed here that although the Kondo effect manifests itself as a resonance channel for the Cooper-pair tunneling, the Kondo peak
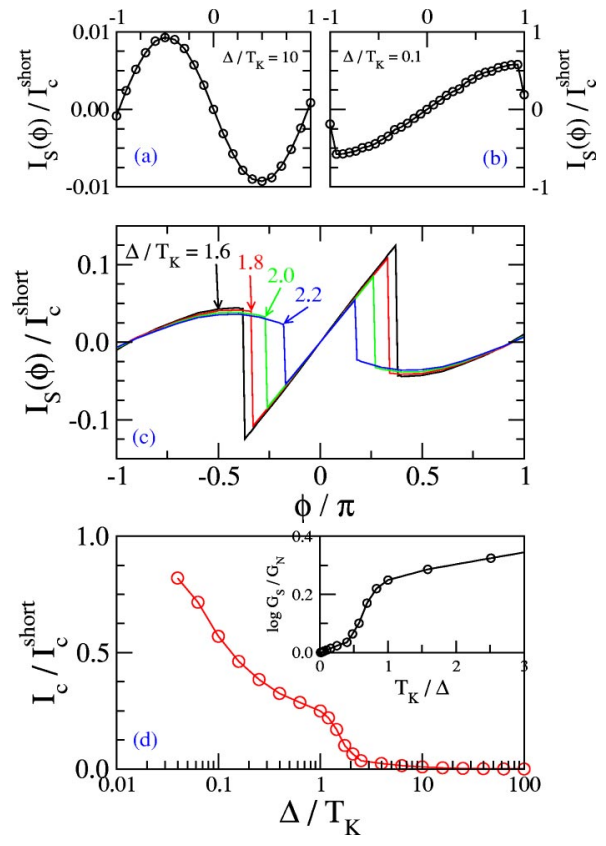

FIG. 3. (Color online) Josephson current $I_{S}(\phi)$ (in units of $I_{c}^{\text {short }} \equiv e \Delta / \hbar$ ) as a function of phase different $\phi$ (a) for $\Delta / T_{K}=10$ and (b) for $\Delta / T_{K}=0.1$. (c) Same curves for $\Delta / T_{K}=1.6,1.8,2.0$, and 2.2 (near the $0-\pi$ junction transition point). (d) Critical current in the Kondo regime. We put $\epsilon_{d}=-U / 2=-0.1 D$ and $\Gamma=0.04 D$. Inset: conductance resulting from the RSJ model (see the text).

of width $T_{K}$ in the quasi-particle excitation spectrum is suppressed (showing a gap) below the energy scale of order $\Delta\left(\ll T_{K}\right)$; see Fig. 2 .

Another interesting regime is the intermediate one $(\Delta$ $\left.\sim T_{K}\right)$. As demonstrated in Fig. 3(c), for $\Delta \sim T_{K}$ the curve of $I_{S}(\phi)$ breaks into three distinct segments. The central segment resembles that of a ballistic short junction, ${ }^{17}$ while the two surrounding segments are parts of a $\pi$-junction curve. ${ }^{6}$ Namely, the critical value $\Delta_{c}(\phi)$ depends on $\phi$ with $\Delta_{c}(\phi)>\Delta_{c}\left(\phi^{\prime}\right)$ for $|\phi|<\left|\phi^{\prime}\right|,{ }^{21}$ for example, $\Delta_{c}(0.3 \pi) \approx 1.6$ and $\Delta_{c}(0) \approx 2.4$. Evidently, the NRG results show that the ground state is a spin singlet in the central segments $\left(\Delta<\Delta_{c}(\phi)\right)$ and a doublet in the other $\left(\Delta>\Delta_{c}\right)$.

\section{EXPERIMENTS}

In the experiments of Buitelaar et al. ${ }^{10}$ the interplay between superconductivity and Kondo physics was observed in non-equilibrium transport (multiple Andreev reflections), 22,23 but no supercurrent was measured. However, the absence of a dissipationless branch in the IV is not surprising in such (intrinsically) small junctions. Indeed thermal or quantum fluctuations in connection with a resistive environment can lead to a finite resistance. ${ }^{24}$ In Ref. 10 the "quality factor" $R_{N} C\left(2 e I_{c} / \hbar C\right)^{1 / 2}$, governing the dynamics of the corresponding resistively-shunted junction (RSJ) model, is always smaller than 1 (Ref. 25) and the junction is therefore overdamped. In this limit the measured resistance $G_{S}$ is directly related to the current-phase relation, roughly like $G_{S} / G_{N}$ $\sim \exp \left(\hbar I_{c} / e T\right) .{ }^{26}$ This enables us to relate our results of Fig. 
3 to the measured crossover of the conductance as function of $T_{K} / \Delta$, see Fig. 4 of Ref. 10. For the experimental temperature $T=50 \mathrm{mK}$ and gap parameter $\Delta \sim 1.2 \mathrm{~K}$, the calculated critical current in Fig. 3(d) means that the factor $\hbar I_{c} / e T$ becomes much larger than 1 in the Kondo regime $\Delta \ll T_{K}$, when the transparent junction limit is reached. Thus, the experimentally observed crossover to $G_{S}>G_{N}$ in this limit is a manifestation of the supercurrent approaching the unitary limit $e \Delta / \hbar$. The inset of Fig. 3(d) shows the conductance as a function of $T_{K} / \Delta$ and that the crossover appears for $T_{K}$ $\approx 0.5 \Delta$, which is in quite good agreement with the experimental result of Ref. 10 .

\section{CONCLUSION}

We have studied the Josephson current and the proximity effect on the QD coupled to two SCs in a whole range of coupling. Our results exhibit a transition from the weak to the strong coupling limit, which occurs when $\Delta \sim T_{K}$. In the weak coupling limit, superconductivity dominates the Kondo physics, and the tunneling of Cooper pairs can be treated perturbatively. The system is a $\pi$-junction, the pairing correlation on the QD is negative, and the ground state is a spin doublet. In the strong coupling limit, the Kondo effect becomes important and manifests itself as a resonance channel for the Cooper-pair tunneling. This leads to a positive Josephson coupling (0-junction) and positive pairing correlation on the QD. Here the Kondo effect in the presence of superconductivity is distinguished from the usual one with normal leads in that the Kondo peak in the quasi-particle excitation spectrum is suppressed completely (exhibiting a gap) for energies below the superconducting gap.

\section{ACKNOWLEDGMENTS}

We thank C. Schönenberger for remarks on Ref. 10 and acknowledge discussions with $\mathrm{C}$. Bruder, M. Gräber, and T. Kontos. This work was supported by the SKORE-A, the eSSC at Postech, the NCCR Nanoscience, and the Swiss NSF.
${ }^{1}$ J. Kondo, Prog. Theor. Phys. 32, 37 (1964).

${ }^{2}$ J. Bardeen, L. N. Cooper, and J. R. Schrieffer, Phys. Rev. 106, 162 (1957); 108, 1175 (1957).

${ }^{3}$ H. Shiba and T. Soda, Prog. Theor. Phys. 41, 25 (1969).

${ }^{4}$ L. I. Glazman and K. A. Matveev, Pis'ma Zh. Tekh. Fiz. 49, 570 (1988) [JETP Lett. 49, 659 (1989)].

${ }^{5}$ B. I. Spivak and S. A. Kivelson, Phys. Rev. B 43, 3740 (1991).

${ }^{6}$ A. V. Rozhkov and D. P. Arovas, Phys. Rev. Lett. 82, 2788 (1999).

${ }^{7}$ A. A. Clerk and V. Ambegaokar, Phys. Rev. B 61, 9109 (2000).

${ }^{8}$ A. V. Rozhkov and D. P. Arovas, Phys. Rev. B 62, 6687 (2000).

${ }^{9}$ E. Vecino, A. Martín-Rodero, and A. Levy Yeyati, Phys. Rev. B 68, 035105 (2003).

${ }^{10}$ M. R. Buitelaar, T. Nussbaumer, and C. Schönenberger, Phys. Rev. Lett. 89, 256801 (2002); M. R. Buitelaar, W. Belzig, T. Nussbaumer, B. Babić, C. Bruder, and C. Schönenberger, Phys. Rev. Lett. 91, 057005 (2003).

${ }^{11}$ D. Goldhaber-Gordon, H. Shtrikman, D. Mahalu, D. AbuschMagder, U. Meirav, and M. A. Kastner, Nature (London) 391, 156 (1998); D. Goldhaber-Gordon, J. Göres, M. A. Kastner, H. Shtrikman, D. Mahalu, and U. Meirav, Phys. Rev. Lett. 81, 5225 (1998); S. M. Cronenwett, T. H. Oosterkamp, and L. P. Kouwenhoven, Science 281, 540 (1998); W. G. van der Wiel, S. De Franceschi, T. Fujisawa, J. M. Elzerman, S. Tarucha, and L. P. Kouwenhoven, ibid. 289, 2105 (2000); Y. Ji, M. Heiblum, D. Sprinzak, D. Mahalu, and H. Shtrikman, Science 290, 779 (2000).

${ }^{12}$ F. D. M. Haldane, Phys. Rev. Lett. 40, 416 (1978).

${ }^{13}$ K. G. Wilson, Rev. Mod. Phys. 47, 773 (1975).

${ }^{14}$ H. R. Krishna-Murthy, J. W. Wilkins, and K. G. Wilson, Phys.
Rev. B 21, 1003 (1980); 21, 1044 (1980).

${ }^{15}$ T. Yoshioka and Y. Ohashi, J. Phys. Soc. Jpn. 69, 1812 (2000).

${ }^{16}$ With $\phi=0$, we map the model in Eqs. (1)-(3) to an effective model with only a single lead, for which the NRG calculation is far more efficient than for the two-lead problem.

${ }^{17}$ C. W. J. Beenakker and H. van Houten, Phys. Rev. Lett. 66, 3056 (1991); A. Furusaki, H. Takayanagi, and M. Tsukada, ibid. 67, 132 (1991).

${ }^{18}$ See also O. Sakai, Y. Shimizu, H. Shiba, and K. Satori, J. Phys. Soc. Jpn. 62, 3181 (1993).

${ }^{19}$ W. Izumida, O. Sakai, and Y. Shimizu, J. Phys. Soc. Jpn. 66, 717 (1997); W. Izumida and O. Sakai, Phys. Rev. B 62, 10260 (2000).

${ }^{20}$ A. V. Rozhkov, D. P. Arovas, and F. Guinea, Phys. Rev. B 64, 233301 (2001).

${ }^{21}$ M.-S. Choi, C. Bruder, and D. Loss, Phys. Rev. B 62, 13569 (2000).

${ }^{22}$ Y. Avishai, A. Golub, and A. D. Zaikin, Phys. Rev. B 63, 134515 (2001).

${ }^{23}$ A. Levy Yeyati, A. Martín-Rodero, and E. Vecino, Phys. Rev. Lett. 91, 266802 (2003).

${ }^{24}$ M. Tinkham, Introduction to Superconductivity (McGraw-Hill, New York, 1996), 2nd ed.

${ }^{25}$ The capacitance of the junction is of the order of $C=100 \mathrm{aF}$. Unfortunately the environmental resistance is not known, but is probably smaller than $h / e^{2}$ since the contact pads are superconducting.

${ }^{26}$ V. Ambegaokar and B. I. Halperin, Phys. Rev. Lett. 22, 1364 (1969). 